Synthesis of Some Heterocyclic Compounds Derived from

1-Aryl-3-(4-hydroxy-3-methoxyphenyl) propene-1-one

Eman Ibrahim AL-Salihi $\quad$ Ahmad Khidr Ahmad

\title{
Synthesis of some heterocyclic compounds derived from 1-Aryl-3-(4- hydroxy-3-methoxyphenyl) propene-1-one
}

\author{
Eman Ibrahim AL-Salihi and Ahmad Khidr Ahmad
}

Chemistry Department - College of Education for Pure Science - Mosul University

Received: 11 September 2015 Accepted: 11 June 2016

\begin{abstract}
$\underline{\text { Abstract }}$
A series of heterocyclic compounds have been synthesized using 1-aryl-3-(4-hydroxy-3methoxypheny) prop-2-ene-1-one (1-4) as starting material which was prepared by claisenschmidt condensation between vanillin and substituted acetophenone.Benzoxazepine derivatives (5-8) and benzodiazepine derivatives (9-12) were synthesized from the reaction of o-aminophenol, o-phenlendiamine with compounds (1-4) respectively. Thereaction of compounds (1-4) with hydrazine hydrate afforded pyrazol derivatives (13-16). While the reaction of compounds (1-4) with hydrazine hydrates in the presences a few drops of glacial acetic acid gave 1- acetyl pyazol derivatives (17-20). Oxirane derivatives (21-24) were synthesized from the reaction of compounds (1-4) with hydrogen peroxide in the basic medium. Reaction of phenylthiourea with compounds (1-4) afforded pyrimidinethion derivative (2527).Finally isoxazol derivatives (28-31) were synthesized by the reaction of compounds (1-4) with hydroxylamine hydrochloride in presences of triethylamine.
\end{abstract}

Key words: vanillin;Pyrazol;Oxirane;Pyrimidinethion;Isoxazol 


\title{
Synthesis of Some Heterocyclic Compounds Derived from
}

1-Aryl-3-(4-hydroxy-3-methoxyphenyl) propene-1-one

Eman Ibrahim AL-Salihi Ahmad Khidr Ahmad

\section{تحضير بعض من المركبات الحلقية غير المتجانسة المشتقة من 1- اريل -3-(4-هيدروكسي-3- ميثوكسيفنيل) بروبين-1-اون}

\author{
إيمان إبراهيم إسماعيل الصالحي و أحمد خضر أحمد الخياط \\ جامعة الموصل - كلية التربية للعلوم الصرفة ـ قسم الكيمياء
}

\section{الخلاصة}

في هذا البحث تم تحضـير 1- أريل-3-(-4 هيدروكسي-3-ميثوكسـي فنيل)- بروب-2-ين-1-اون (4-1) عن طريق تكاثف كليزن- شمدت بين الفانيلينومعوضـات الاسيتوفينون.حضرت معوضـات بنزو اكسازبين( 8-5) من مفاعله المركبات(4-1-1) مع أورثو -أمينو فينول.مشتقات البنزوثنائي أزين (12-9) حضرت من تفاعل المركبات (4-1) مع أورثوفنيلين ثنائي الأمين ـ تفاعل المركبات (4-1) مع الهيد رازين أعط مشــتقات الباير ازول (16-13) ، بينما تفاعل المركبات (4-1) مع الهيد

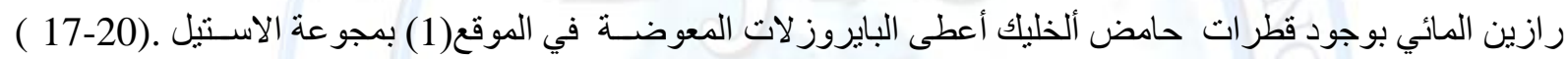
وكذللك تم تحضير معوضـات الاوكسيران (21-24) من تفاعل المركبات (4-1) مع 30\% بيرو كسيد الهيدروجين . تفاعل

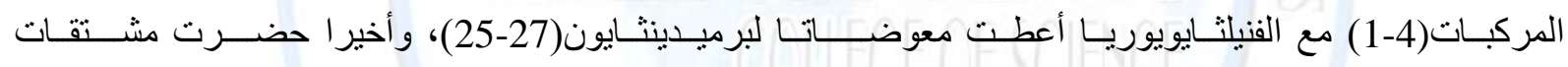
الايزواوكسـازول(31-28) عن طريق مفاعله المركبات (4-1) مع الهيدروكسـيل أمين هيدروكلوريد شـخصـت المركبات المحضرة بالطرق الطيفية و الفيزياويه المتاحة.

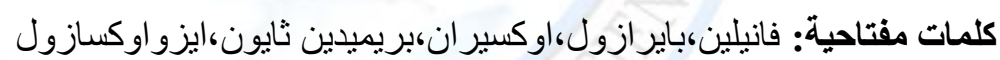

\section{Introduction}

The chemistry of chalcone characteristic by extensive scientific studies by a number researchers, some of these chalcone synthesis by the reaction of acetophenone with substituted aldehyde ${ }^{(1)}$ othersreseacherersuse micro wave technology for preparation of a number of these derivatives ${ }^{(2)}$. This type or compounds encouraged us to synthesize a new heterocyclic derived from these compounds. Rana and Singh have synthesized pyarzole-1-ethanone derivatives depending on chalcone as starting material ${ }^{(2)}$, many pyrazoline and isoxazole and pyrimidinone derivatives were synthesized depending on chalcone derivatives as starting material. ${ }^{(3-4)}$ Hetrocycliccompounds have dispelled an impressive array of biological activity among which anti-malarial $^{(5)}$, anti-protozoa ${ }^{(6)}$, anti-Inflammatory ${ }^{(7)}$ and immunlodulatory ${ }^{(8)}$. 


\section{Synthesis of Some Heterocyclic Compounds Derived from \\ 1-Aryl-3-(4-hydroxy-3-methoxyphenyl) propene-1-one}

\section{Eman Ibrahim AL-Salihi $\quad$ Ahmad Khidr Ahmad}

In this paper chalcone derivatives was used as important starting materials for the synthesis of various classes of heterocyclic compounds such as isoxazole, pyrazolines, substituted pyrazolines and pyrimidinone derivative. Many1, 5-benzoxazepines and benzodiazepine derivative were also prepared.

\section{Experimental}

Melting points were measured on Electro thermal Melting Point Apparatus and are uncorrected. The IR spectra were recorded by using infrared spectrophotometer model Tensor 27 Bruckner Co. Germany. Ultra violet light recorded by Shimadzu UV-210 Double-Beam Spectrophotometer in DMF as solvent.

General procedure for synthesis of 1-aryl-3-(4-hydroxy-3-methoxyphenyl) prop-2-ene-1one $^{(9)}(1-4)$.

A mixture of $(1.52 \mathrm{~g}, 0.01 \mathrm{~mol})$ vanillin, $(0.01 \mathrm{~mol})$ substituted acetophenone in $20 \mathrm{ml}$ absolute ethanol and 10 drops of thionyl chloride were stirring for $10 \mathrm{hr}$., and then refluxed for $6 \mathrm{hr}$. The solvent was evapored under reduce pressure crush ice was added to the precipitate and left in cool condition overnight, the precipitate separated by filtration, dried and recrystallized from ethanol, The physical properties and spectral data were listed in Tables $(1,9)$.

\section{2-Aryl-4-(4-hydroxy-3-meyhoxyphenyl) -2, 3-dihydro-1H-1, 5-benzoxazepine ${ }^{(10)}(5-8)$}

A mixture of the apreroppratechalcone derivative (1-4) $(0.025 \mathrm{~mol})$, o- aminophenol $(0.025 \mathrm{~mol}$, $2.74 \mathrm{~g}$ ) in $20 \mathrm{ml}$ methanol and a few drops from glacial acetic acid was refluxed for $8 \mathrm{hr}$, the solvent was evaporated under reduced pressure, and the residue was recrystallized from dichloromethane. The physical properties and spectral data were listed in Tables $(2,10)$

\section{4- Aryl-2-(4-hydroxy-3-methoxyphenyl) -2, 3-dihydro-1H-1, 5-benzodiazepine ${ }^{(11)}(9-12)$.}

A mixture of the appropriate chalcone derivative (1-4) (0.02mol), ophenylendiamine $(0.02 \mathrm{~mol}, 2.16 \mathrm{~g})$ in absolute ethanol $20 \mathrm{ml}$ and a few drops of glacial acetic acid was refluxed for 12 hrs., the solvent was removed under reduce pressure, the obtained 


\section{Synthesis of Some Heterocyclic Compounds Derived from \\ 1-Aryl-3-(4-hydroxy-3-methoxyphenyl) propene-1-one}

Eman Ibrahim AL-Salihi

Ahmad Khidr Ahmad

precipitate was recrystallized from dry ethanol .Some physical properties and spectral data were listed in Tables $(3,11)$

\section{3- Aryl-5-(4-hydroxy-3-melthoxyphenyl) -4, 5-dihydro-1H-pyrazole ${ }^{(12)}(13-16)$}

A mixture of the appropriate chalcone derivative $(1-4)(0.004 \mathrm{~mol}),(0.025 \mathrm{~mol}, 1.25 \mathrm{~g})$ hydrazine hydrate in $20 \mathrm{ml}$ glacial acetic acid was refluxed for $8 \mathrm{hr}$, then ice-water added and left to cool box overnight the precipitate was filtered and recrystallized from ethanol. The physical properties and spectral data were indicated in Tables $(4,12)$

\section{1-acetyl-3-aryl-5-(4-hydroxy-3-methoxyphenyl) -4, 5-dihydro-1H-pyrazole ${ }^{(13,14)}(17-20)$}

A mixture of the appropriate chalcone derivative (1-4) $(0.01 \mathrm{~mol})$ in $20 \mathrm{ml}$ absolute ethanol, hydrazine hydrate $(0.01 \mathrm{~mol}, 1 \mathrm{~g})$ and a few drops of glacial acetic acid were refluxed for $9 \mathrm{hr}$. the excess of solvent was removed, and the product washed with water then recrystallized from ethanol. The physical properties and spectral data were listed in Tables $(5,13)$

\section{2-Aroyl 3-(4-hydroxy-3-methoxyphenyl)-oxariane ${ }^{(15)}$ (21-24)}

A mixture of $1 \mathrm{ml}(10 \%) \mathrm{NaOH}, 10 \mathrm{ml}$ ( $30 \%$ ) hydrogen peroxide was added tothe appropriate chalcone derivative $(1-4)(0.01 \mathrm{~mol})$ in $10 \mathrm{ml}$ ethanol, the reaction mixture was stirred at room temperature for $14 \mathrm{hr}$., the mixture was neutralized by $\mathrm{HCl}$ and left at room temperature for 24 hr. the formed precipated was filtered and recrystallized from ethanol. The physical properties and spectral data were indicated in Tables $(6,14)$

\section{6- Aryl-4-(4-hydroxy-3-methoxy phenyl)-3-phenyl-3, 4-dihydro pyrimidine-2-(1H) - thione $^{(16)}(25-27)$}

A mixture of the appropriate chalcone derivative (1-4) (0.006mol) in $10 \mathrm{ml}$ absolute ethanol and $(0.46 \mathrm{~g})$ sodium metal dissolved in absolute ethanol was added to the a solution of phenylthiourea $(0.006 \mathrm{~mol}, 0.76 \mathrm{~g})$ in absolute ethanol. The final solution was refluxed for $10 \mathrm{hr}$.Cool, water was then added. The formed precipitate was separated by filtration and then crystallized from ethanol. Some physical and spectral data were indicated in Tables $(7,15)$.

3-aryl-5-(4-hydroxy-3-methoxy phenyl) -4, 5-dihydro isoxazole ${ }^{(17)}(28-31)$

A mixture of the appropriatechalcone derivative (1-4) $(0.023 \mathrm{~mol})$, hydroxyl amine hydrochloride $(0.023 \mathrm{~mol}, 1.59 \mathrm{~g})$ and $0.4 \mathrm{~g}$ g sodium hydroxide in $30 \mathrm{ml}$ ethanol was refluxed 


\section{Synthesis of Some Heterocyclic Compounds Derived from \\ 1-Aryl-3-(4-hydroxy-3-methoxyphenyl) propene-1-one}

Eman Ibrahim AL-Salihi
Ahmad Khidr Ahmad

for $7 \mathrm{hr}$., the solvent was removed, and the resultant was poured into ice-water with vigorous stirring, then it was kept in cool box for $24 \mathrm{hr}$. Then the solid product was filtrated and recrystallized from dioxane. Some physical and spectral data were indicated in Tables $(8,16)$

\section{$\underline{\text { Result and Discussion }}$}

The 1-aryl-3-(4-hydroxy-3-methoxy phenyl) propen-1-one (1-4) were obtained from refluxing vanillin with substituted acetophenone in absolute ethanol in presences of a few drops of thionyl chloride. These compounds were identified by IR which exhibits characteristic bands in the following range. The bands at $\left(1654-1683 \mathrm{~cm}^{-1}\right)^{(18)}$ were assigned for the carboxyl group stretching. While the bands at $\left(3062-3184 \mathrm{~cm}^{-1}\right)$ related to the $\left(\mathrm{C}-\mathrm{H}_{\mathrm{Ar}}\right)$ stretching and bands at $\left(1573-1653 \mathrm{~cm}^{-1}\right)$ for $(\mathrm{C}=\mathrm{C})$ groups. The spectrum also showed bands at range $\left(3315-3525 \mathrm{~cm}^{-}\right.$ $\left.{ }^{1}\right)$ for the $(\mathrm{OH})^{(19)}$ group, as shown in table (9). 1, 5-benzoxapine derivatives (5-8) were prepared by the reaction of appropriate chalcone with o-amino phenol. The product was identified by the stretching bands at $\left(1609-1655 \mathrm{~cm}^{-1}\right)$ for the $(\mathrm{C}=\mathrm{N})$ group, also bands at $\left(1124-1160 \mathrm{~cm}^{-1}\right) \&$ $\left(1245-1285 \mathrm{~cm}^{-1}\right)$ for symmetrical asymmetrical(C-O-C $)^{(20)}$ respectively. The stretching absorption bands at $\left(3435-3566 \mathrm{~cm}^{-1}\right)$ for $(\mathrm{O}-\mathrm{H})$ groups. Also the spectrum characteristic bythe disappearance of the bands for $(\mathrm{C}=\mathrm{C})$ and $(\mathrm{C}=\mathrm{O})$ groups as shown in Table (10).

The reaction of chalcone derivative with o.phenylenediamine afforded benzodiazepine derivatives (9-12) which were identified by IR spectrum through the appearance of the following stretching bands; at $\left(1609-1655 \mathrm{~cm}^{-1}\right)$ for $\mathrm{C}=\mathrm{N}$ group, $\left(3118-3287 \mathrm{~cm}^{-1}\right)$ for $\mathrm{N}-\mathrm{H}$ stretching, while (3275-3367 $\mathrm{cm}^{-1}$ ) for O-H stretching and absorption band within the range ( $1241-1277 \mathrm{~cm}^{-1}$ ) due to $\mathrm{C}-\mathrm{N}$ stretching, The spectrum was also characterized by the absence of $\mathrm{C}=\mathrm{C}$ and $\mathrm{C}=\mathrm{O}$ groups.

Condensation of ofchalcone derivatives with hydrazine hydrate in glacial acetic acid as solvent afforded pyrazole derivatives (13-16) which were identified by IR. These spectra showed the absence of stretching banding of the carbonyl, $\mathrm{C}=\mathrm{C}$ groups and the appearances of new bands at $\left(1605-1695 \mathrm{~cm}^{-1}\right)$ for $\mathrm{C}=\mathrm{N}$ stretching vibration and bands absorbed at $\left(3166-3332 \mathrm{~cm}^{-1}\right)$ and $\left(3524-3584 \mathrm{~cm}^{-1}\right)^{(21)}$ for N-H and O-H respectively, also N-N stretching vibration was absorbed at $\left(1020-1033 \mathrm{~cm}^{-1}\right)$ as shown in Table (12) 


\section{Synthesis of Some Heterocyclic Compounds Derived from 1-Aryl-3-(4-hydroxy-3-methoxyphenyl) propene-1-one}

\section{Eman Ibrahim AL-Salihi}

\section{Ahmad Khidr Ahmad}

The condensation of compounds (1-4) with hydrazine hydrate in absolute ethanol in the presence of a few drops of glacial acetic acid afforded 1-acetylpyrazol derivatives (17-20) which were identified by IR. The appearance of bands at $\left(1506-1596 \mathrm{~cm}^{-1}\right)$ for the $\mathrm{C}=\mathrm{N}^{(22)}$ stretching vibration. Bands at (1426-1507 $\mathrm{cm}^{-1)}$ for $\mathrm{N}-\mathrm{N}$ stretching vibration band. The spectrum also showed band at $\left(3612-3499 \mathrm{~cm}^{-1}\right)$ for O-H,also FT-IR indicated by the appearance of band at (1596-1646 $\left.\mathrm{cm}^{-1}\right)$ for carbonyl group, as well as the disappearance of stretching bands related to $\mathrm{C}=\mathrm{C}$ group, table(13). Shows the spectral data of these compounds.

Compounds (21-24) were identified by the appearance of the following bands; at (1640-1653 $\mathrm{cm}^{-1}$ ) for carbonyl group , bands at $\left(1076-1168 \mathrm{~cm}^{-1}\right) \&\left(1278-1285 \mathrm{~cm}^{-1}\right)$ for symmetrical and asymmetrical (C-O-C) bonds stretching respectively and the band absorbed within the range (3177-3669 $\mathrm{cm}^{-1}$ ) due to the $\mathrm{O}-\mathrm{H}$ stretching, also the spectrum characterized by the absent of band for $\mathrm{C}=\mathrm{C}$ group. Table (14) shows some spectral data of these compounds.

The reaction of chalconederivatives (1-4) with phenylthiourea offored pyrimidine thione derivatives Table (15) which shows absorption bands of compounds (25-27) at (1551- 1673 $\left.\mathrm{cm}^{-1}\right)$ attributed to the stretching vibration of $\mathrm{C}=\mathrm{C}$ group ${ }^{(23)}$. Also the bands at $\left(1115-1166 \mathrm{~cm}^{-}\right.$ ${ }^{1}$ ) attributed to the $\mathrm{C}=\mathrm{S}$ group. While the stretching vibration of $\mathrm{O}-\mathrm{H}$ group appeared at (3347$3596 \mathrm{~cm}^{-1}$ ), also the spectrum shows the absence of carbonyl groups.

The IR spectra for isoxazole compounds (28-31) shows absorption bands at (1614-1654 $\mathrm{cm}^{-1)}$ attributed to the stretching vibration of $\mathrm{C}=\mathrm{N}$. The spectra were also showed absorption bands at $\left(1106-1091 \mathrm{~cm}^{-1}\right) \&\left(1265-1272 \mathrm{~cm}^{-1}\right)$ for symmetrical and asymmetrical C-O-C bonds stretching, while the bands at $\left(750-784 \mathrm{~cm}^{-1}\right)$ attributed to the $\mathrm{N}-\mathrm{O}$ bonds. Also the spectrum shows the absent of $\mathrm{C}=\mathrm{C}$ group. Table (16) shows some spectral data of these compounds. 
Synthesis of Some Heterocyclic Compounds Derived from 1-Aryl-3-(4-hydroxy-3-methoxyphenyl) propene-1-one

Eman Ibrahim AL-Salihi

Ahmad Khidr Ahmad

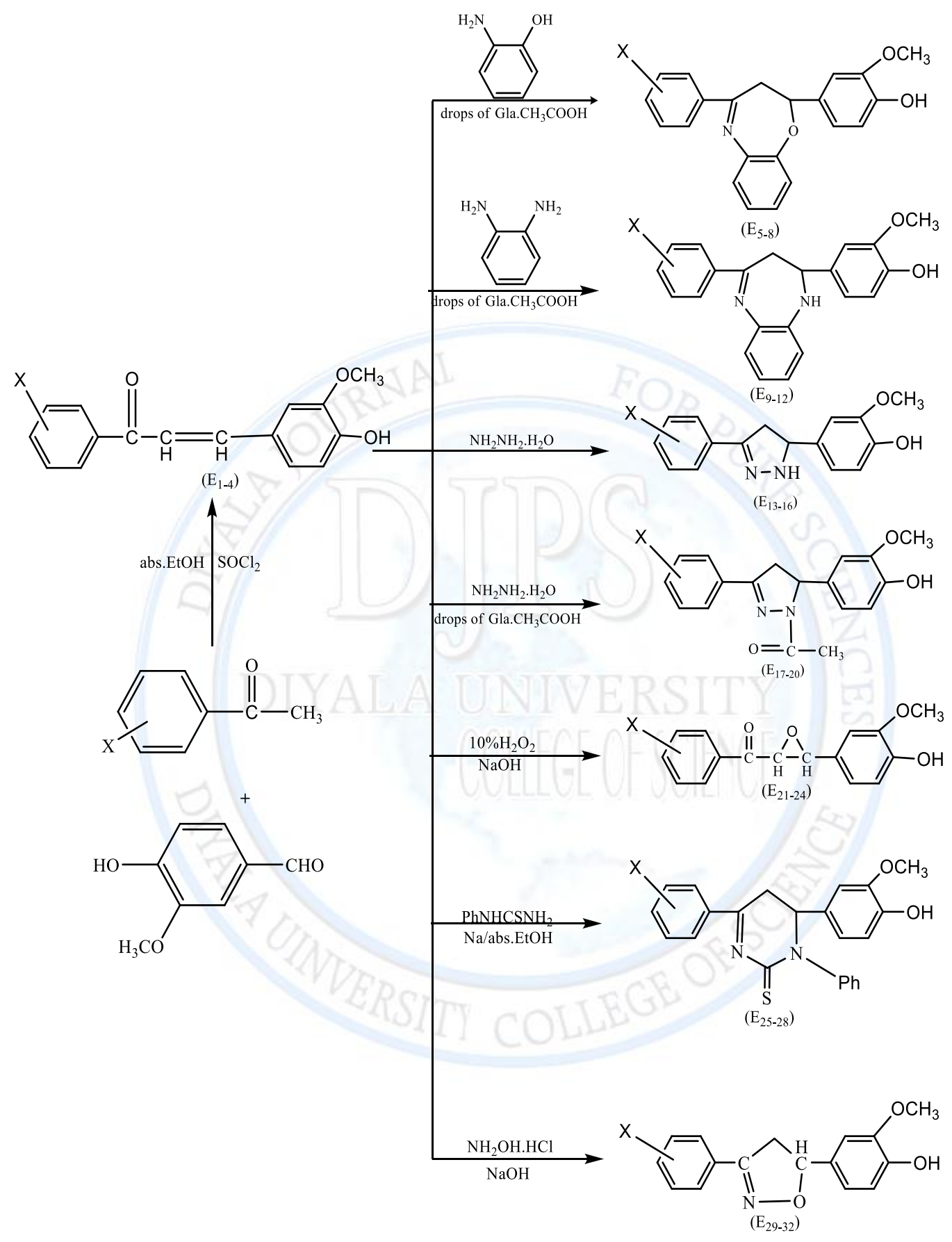

$\mathrm{X}=\mathrm{H}, 4-\mathrm{NO} 2,2-\mathrm{Cl}, 4-\mathrm{OCH}_{3}$,

Scheme for general reactions 


\section{DIYALA JOURNAL FOR PURE SCIENCES}

Synthesis of Some Heterocyclic Compounds Derived from

1-Aryl-3-(4-hydroxy-3-methoxyphenyl) propene-1-one

Eman Ibrahim AL-Salihi Ahmad Khidr Ahmad

Table (1): Some physical constant for compounds (1-4)<smiles>[X]c1cccc(C(=O)C=Cc2ccc(O)c(OC)c2)c1</smiles>

\begin{tabular}{|c|c|c|c|c|c|}
\hline Comp. No & $\mathrm{X}$ & Molecular Formula & M.P $\left(\mathrm{C}^{\circ}\right)$ & Yield (\%) & Color \\
\hline 1 & $\mathrm{H}$ & $\mathrm{C}_{16} \mathrm{H}_{14} \mathrm{O}_{3}$ & $70-72$ & 64 & Green \\
\hline 2 & $2-\mathrm{Cl}$ & $\mathrm{C}_{16} \mathrm{H}_{13} \mathrm{ClO}_{3}$ & $117-119$ & 79 & $\begin{array}{c}\text { Yellowish- } \\
\text { green }\end{array}$ \\
\hline 3 & $\mathrm{OCH}_{3}$ & $\mathrm{C}_{17} \mathrm{H}_{16} \mathrm{O}_{4}$ & $\begin{array}{c}131-133 \\
(130)^{(24)}\end{array}$ & 74 & Pale yellow \\
\hline 4 & $4-\mathrm{NO}_{2}$ & $\mathrm{C}_{16} \mathrm{H}_{13} \mathrm{NO}_{5}$ & $62-63$ & 80 & $\begin{array}{c}\text { Yellowish } \\
\text { orange }\end{array}$ \\
\hline
\end{tabular}

Table (2): Some physical constant for compounds (5-8)

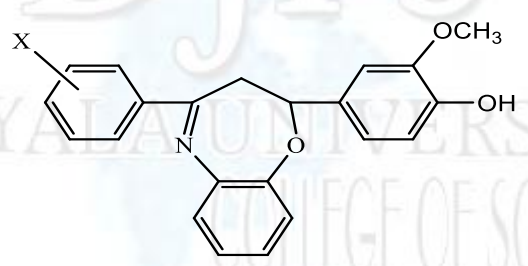

\begin{tabular}{|c|c|c|c|c|c|}
\hline Comp. No. & $\mathrm{X}$ & Molecular Formula & M. P. $\left({ }^{\circ} \mathrm{C}\right)$ & Yield (\%) & Color \\
\hline 5 & $4-\mathrm{OCH}_{3}$ & $\mathrm{C}_{23} \mathrm{H}_{19} \mathrm{NO}_{4}$ & $140-142$ & 88 & Green \\
\hline 6 & $4-\mathrm{NO}_{2}$ & $\mathrm{C}_{22} \mathrm{H}_{16} \mathrm{~N}_{2} \mathrm{O}_{5}$ & $146-147$ & 78 & Dark yellow \\
\hline 7 & $\mathrm{H}$ & $\mathrm{C}_{22} \mathrm{H}_{16} \mathrm{NO}_{3}$ & $144-146$ & 80 & Brown \\
\hline 8 & $2-\mathrm{Cl}$ & $\mathrm{C}_{22} \mathrm{H}_{15} \mathrm{NO}_{3}$ & $48-50$ & 68 & Yellow \\
\hline
\end{tabular}


Synthesis of Some Heterocyclic Compounds Derived from

1-Aryl-3-(4-hydroxy-3-methoxyphenyl) propene-1-one

Eman Ibrahim AL-Salihi Ahmad Khidr Ahmad

Table (3): Some physical constant for compounds (9-12)

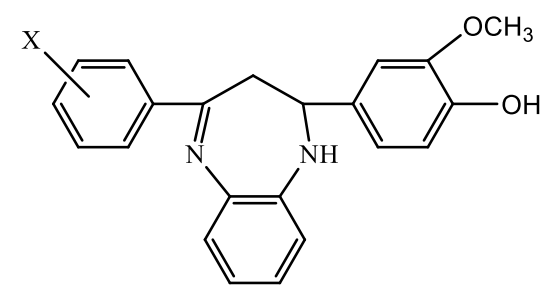

\begin{tabular}{|c|c|c|c|c|c|}
\hline Comp. No. & $\mathrm{X}$ & Molecular Formula & M. P. $\left({ }^{\circ} \mathrm{C}\right)$ & Yield (\%) & Color \\
\hline 9 & $4-\mathrm{CH}_{3}$ & $\mathrm{C}_{23} \mathrm{H}_{20} \mathrm{~N}_{2} \mathrm{O}_{3}$ & $130-132$ & 78 & Orange \\
\hline 10 & $4-\mathrm{NO}_{2}$ & $\mathrm{C}_{23} \mathrm{H}_{16} \mathrm{~N}_{3} \mathrm{O}_{4}$ & $106-107$ & 88 & Yellow \\
\hline 11 & $\mathrm{H}$ & $\mathrm{C}_{22} \mathrm{H}_{18} \mathrm{~N}_{2} \mathrm{O}_{2}$ & $140-142$ & 70 & Green \\
\hline 12 & $2-\mathrm{Cl}$ & $\mathrm{C}_{22} \mathrm{H}_{18} \mathrm{~N}_{2} \mathrm{O}_{2} \mathrm{Cl}$ & $98-99$ & 78 & Brown \\
\hline
\end{tabular}

Table (4): Some physical constant for compounds (13-16)<smiles>[X]c1cccc(C2=NNC(c3ccc(O)c(OC)c3)C2)c1</smiles>

\begin{tabular}{|c|c|c|c|c|c|}
\hline Comp. No & $\mathrm{R}$ & Molecular Formula & $\mathrm{M} . \mathrm{P}\left(\mathrm{C}^{\mathrm{o}}\right)$ & Yield (\%) & Color \\
\hline 13 & $\mathrm{H}$ & $\mathrm{C}_{16} \mathrm{H}_{14} \mathrm{~N}_{2} \mathrm{O}_{2}$ & $198-200$ & 94 & Dark yellow \\
\hline 14 & $2-\mathrm{Cl}$ & $\mathrm{C}_{16} \mathrm{H}_{14} \mathrm{~N}_{2} \mathrm{O}_{2} \mathrm{Cl}$ & $126-127$ & 78 & White \\
\hline 15 & $4-\mathrm{OCH}_{3}$ & $\mathrm{C}_{17} \mathrm{H}_{16} \mathrm{~N}_{2} \mathrm{O}_{3}$ & $157-159$ & 84 & Brown \\
\hline $\mathrm{E}_{44}$ & $4-\mathrm{NO}_{2}$ & $\mathrm{C}_{16} \mathrm{H}_{13} \mathrm{~N}_{3} \mathrm{O}_{4}$ & $166-170$ & 63 & Yellow \\
\hline
\end{tabular}

Table (5): Some physical constant for compounds (17-20)

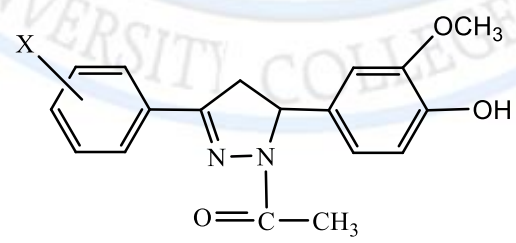

\begin{tabular}{|c|c|c|c|c|c|}
\hline Comp. No & $\mathrm{X}$ & Molecular Formula & $\mathrm{M} . \mathrm{P}\left(\mathrm{C}^{\circ}\right)$ & Yield (\%) & Color \\
\hline 17 & $\mathrm{H}$ & $\mathrm{C}_{18} \mathrm{H}_{16} \mathrm{~N}_{2} \mathrm{O}_{3}$ & $46-47$ & 70 & Yellow \\
\hline 18 & $4-\mathrm{NO}_{2}$ & $\mathrm{C}_{19} \mathrm{H}_{15} \mathrm{~N}_{3} \mathrm{O}_{5}$ & $193-195$ & 77 & Orange \\
\hline 19 & $4-\mathrm{OCH}_{3}$ & $\mathrm{C}_{19} \mathrm{H}_{18} \mathrm{~N}_{2} \mathrm{O}_{4}$ & $75-76$ & 68 & Green \\
\hline 20 & $2-\mathrm{Cl}$ & $\mathrm{C}_{18} \mathrm{H}_{16} \mathrm{~N}_{2} \mathrm{O}_{3} \mathrm{Cl}$ & $130-131$ & 65 & White \\
\hline
\end{tabular}


Synthesis of Some Heterocyclic Compounds Derived from

1-Aryl-3-(4-hydroxy-3-methoxyphenyl) propene-1-one

Eman Ibrahim AL-Salihi Ahmad Khidr Ahmad

Table (6): Some physical constant for compounds (21-24)

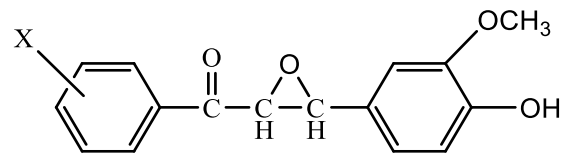

\begin{tabular}{|c|c|c|c|c|c|}
\hline Comp. No. & $\mathrm{X}$ & $\begin{array}{c}\text { Molecular } \\
\text { Formula }\end{array}$ & $\begin{array}{c}\text { M. P. } \\
\left({ }^{\circ} \mathrm{C}\right)\end{array}$ & $\begin{array}{c}\text { Yield } \\
(\%)\end{array}$ & Color \\
\hline 21 & $4-\mathrm{NO} 2$ & C15H13NO6 & $163-165$ & 78 & Yellow \\
\hline 22 & $4-\mathrm{OCH} 3$ & $\mathrm{C} 17 \mathrm{H} 15 \mathrm{O} 5$ & $80-82$ & 70 & Pale yellow \\
\hline 23 & $2-\mathrm{Cl}$ & $\mathrm{C} 15 \mathrm{H} 13 \mathrm{O} 4 \mathrm{Cl}$ & $70-71$ & 60 & Pink \\
\hline 24 & $\mathrm{H}$ & $\mathrm{C} 15 \mathrm{H} 13 \mathrm{O} 4$ & $170-172$ & 65 & Green yellow \\
\hline
\end{tabular}

Table (7): Some physical constant for compounds (25-28)

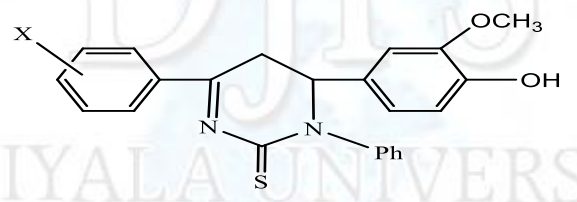

\begin{tabular}{|c|c|c|c|c|c|}
\hline Comp. No. & $\mathrm{X}$ & Molecular Formula & M. P. $\left({ }^{\circ} \mathrm{C}\right)$ & Yield (\%) & Color \\
\hline 25 & $4-\mathrm{OCH} \mathrm{H}_{3}$ & $\mathrm{C}_{24} \mathrm{H}_{20} \mathrm{~N}_{2} \mathrm{O}_{3} \mathrm{~S}$ & $287-289 \mathrm{~d}$ & 84 & Orange yellow \\
\hline 26 & $\mathrm{H}$ & $\mathrm{C}_{23} \mathrm{H}_{18} \mathrm{~N}_{2} \mathrm{O}_{2} \mathrm{~S}$ & $303-304$ & 89 & Orange \\
\hline 27 & $2-\mathrm{Cl}$ & $\mathrm{C}_{23} \mathrm{H}_{17} \mathrm{~N}_{2} \mathrm{O}_{2} \mathrm{SCl}$ & $75-77$ & 80 & Brown \\
\hline 28 & $4-\mathrm{NO}_{2}$ & $\mathrm{C}_{23} \mathrm{H}_{17} \mathrm{~N}_{3} \mathrm{O}_{4} \mathrm{~S}$ & $96-97$ & 78 & Reed \\
\hline
\end{tabular}

Table (8): Some physical constant for compounds (29-31)

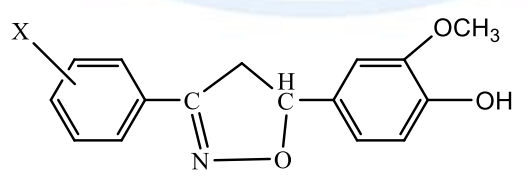

\begin{tabular}{|c|c|c|c|c|c|}
\hline Comp. No. & $\mathrm{X}$ & Molecular Formula & M. P. $\left({ }^{\circ} \mathrm{C}\right)$ & Yield (\%) & Color \\
\hline 29 & $4-\mathrm{OCH}_{3}$ & $\mathrm{C}_{17} \mathrm{H}_{15} \mathrm{NO}_{3}$ & $278-280$ & 68 & Leady \\
\hline 30 & $4-\mathrm{NO}_{2}$ & $\mathrm{C}_{16} \mathrm{H}_{12} \mathrm{~N}_{2} \mathrm{O}_{5}$ & $170-171$ & 70 & Orang \\
\hline 31 & $2-\mathrm{Cl}$ & $\mathrm{C}_{16} \mathrm{H}_{13} \mathrm{ClNO}_{3}$ & $78-80$ & 53 & White \\
\hline 32 & $\mathrm{H}$ & $\mathrm{C}_{16} \mathrm{H}_{13} \mathrm{NO}_{3}$ & $98-99$ & 50 & Brown \\
\hline
\end{tabular}




\section{DIVALA JOURNAL FOR PURE SCIENCES}

\section{Synthesis of Some Heterocyclic Compounds Derived from}

1-Aryl-3-(4-hydroxy-3-methoxyphenyl) propene-1-one

Eman Ibrahim AL-Salihi Ahmad Khidr Ahmad

Table (9): IR spectral data for compounds (1-4)

\begin{tabular}{|c|c|c|c|c|c|c|c|}
\hline \multirow{2}{*}{ Comp no. } & \multirow{2}{*}{$\mathrm{X}$} & $\mathrm{O}-\mathrm{H}$ & $(\mathrm{C}-\mathrm{H}) \mathrm{Ar}$ & $\mathrm{C}=\mathrm{O}$ & $\mathrm{C}=\mathrm{C}$ & $\mathrm{C}=\mathrm{C}_{\mathrm{Ar}}$ & Other \\
\hline 1 & $\mathrm{H}$ & 3315 & 3062 & 1683 & 1653 & 1581 & $\begin{array}{c}\mathrm{As}(\mathrm{C}-\mathrm{O}-\mathrm{C})(1168) \\
\mathrm{Sy}(\mathrm{C}-\mathrm{O}-\mathrm{C})(1035)\end{array}$ \\
\hline 2 & $2-\mathrm{Cl}$ & 3318 & 3184 & 1654 & 1590 & 1557 & $\begin{array}{c}\mathrm{As}(\mathrm{C}-\mathrm{O}-\mathrm{C})(1348) \\
\mathrm{Sy}(\mathrm{C}-\mathrm{O}-\mathrm{C})(1082)\end{array}$ \\
\hline 3 & $4-\mathrm{OCH}_{3}$ & 3420 & 3076 & 1670 & 1650 & 1583 & $\begin{array}{c}\mathrm{As}(\mathrm{C}-\mathrm{O}-\mathrm{C})(1239) \\
\mathrm{Sy}(\mathrm{C}-\mathrm{O}-\mathrm{C})(1021)\end{array}$ \\
\hline 4 & $4-\mathrm{NO}_{2}$ & 3525 & 3107 & 1678 & 1573 & 1573 & $\begin{array}{c}\mathrm{As}(\mathrm{C}-\mathrm{O}-\mathrm{C})(1261) \\
\mathrm{Sy}(\mathrm{C}-\mathrm{O}-\mathrm{C})(1076)\end{array}$ \\
\hline
\end{tabular}

Table (10): IR spectral data for compounds (5-8)

\begin{tabular}{|c|c|c|c|c|c|c|c|c|c|}
\hline \multirow{3}{*}{$\begin{array}{c}\text { Comp } \\
\text { no. }\end{array}$} & \multirow{3}{*}{$X$} & \multicolumn{7}{|c|}{ IR v cm-1 (KBr) } & \multirow{3}{*}{ Other } \\
\hline & & \multirow{2}{*}{$\mathrm{N}-\mathrm{H}$} & \multirow{2}{*}{$\mathrm{O}-\mathrm{H}$} & \multirow{2}{*}{$\mathrm{C}-\mathrm{H}_{\mathrm{Ar}}$} & \multirow{2}{*}{$\mathrm{C}=\mathrm{N}$} & \multirow{2}{*}{$\mathrm{C}=-\cdots \mathrm{C}$} & \multicolumn{2}{|c|}{$\mathrm{C}-\mathrm{O}-\mathrm{C}$} & \\
\hline & & & & & & & As & Sy & \\
\hline 5 & $4-\mathrm{OCH}_{3}$ & 3204 & 3566 & 3007 & 1649 & 1584 & 1261 & 1154 & $E$ \\
\hline 6 & $4-\mathrm{NO}_{2}$ & 3435 & 3535 & 3076 & 1650 & 1558 & 1253 & 1124 & $\begin{array}{l}\mathrm{As}\left(\mathrm{C}-\mathrm{NO}_{2}\right)(1505) \\
\mathrm{Sy}\left(\mathrm{C}-\mathrm{NO}_{2}\right)(1341)\end{array}$ \\
\hline 7 & $\mathrm{H}$ & 3385 & 3493 & 3114 & 1645 & 1556 & 1285 & 1136 & $\sigma^{2}$ \\
\hline 8 & $2-\mathrm{Cl}$ & 3480 & 3522 & 3076 & 1693 & 1580 & 1245 & 1160 & $\mathrm{C}-\mathrm{Cl}(746)$ \\
\hline
\end{tabular}

Table (11): IR spectral data for compounds (9-12)

\begin{tabular}{|c|c|c|c|c|c|c|c|c|}
\hline \multirow{2}{*}{$\begin{array}{c}\text { Comp } \\
\text { no. }\end{array}$} & \multirow[t]{2}{*}{$\mathrm{X}$} & \multicolumn{7}{|c|}{ IR $v \mathrm{~cm}^{-1}(\mathrm{KBr})$} \\
\hline & & $\mathrm{N}-\mathrm{H}$ & $\mathrm{O}-\mathrm{H}$ & $(\mathrm{C}-\mathrm{H})_{\mathrm{Ar}}$ & $\mathrm{C}=\mathrm{N}$ & $\mathrm{C}=\mathrm{C}_{\mathrm{Ar}}$ & $\mathrm{C}-\mathrm{N}$ & Other \\
\hline 9 & $4-\mathrm{OCH}_{3}$ & 3287 & 3367 & 3075 & 1649 & 1566 & 1277 & \\
\hline 10 & $4-\mathrm{NO}_{2}$ & 3236 & 3360 & 3093 & 1646 & 1558 & 1265 & $\begin{array}{l}\mathrm{As}_{\left(\mathrm{C}-\mathrm{NO}_{2}\right)}(1505) \\
\mathrm{Sy}\left(\mathrm{C}-\mathrm{NO}_{2}\right)(1341)\end{array}$ \\
\hline 11 & $\mathrm{H}$ & 3118 & 3366 & 3053 & 1609 & 1514 & 1270 & \\
\hline 12 & $2-\mathrm{Cl}$ & 3189 & 3275 & 3073 & 1655 & 1552 & 1241 & $(\mathrm{C}-\mathrm{Cl})(739)$ \\
\hline
\end{tabular}




\section{DIYALA JOURNAL FOR PURE SCIENCES}

Synthesis of Some Heterocyclic Compounds Derived from

1-Aryl-3-(4-hydroxy-3-methoxyphenyl) propene-1-one

Eman Ibrahim AL-Salihi Ahmad Khidr Ahmad

Table (12): IR spectral data for compounds (13-16)

\begin{tabular}{|c|c|c|c|c|c|c|c|c|c|c|}
\hline \multirow{3}{*}{$\begin{array}{c}\text { Comp } \\
\text { no. }\end{array}$} & \multirow{3}{*}{$\mathrm{X}$} & \multicolumn{9}{|c|}{ IR $v \mathrm{~cm}^{-1}(\mathrm{KBr})$} \\
\hline & & \multirow{2}{*}{$\mathrm{O}-\mathrm{H}$} & \multirow{2}{*}{$\mathrm{N}-\mathrm{H}$} & \multirow{2}{*}{$\begin{array}{c}\mathrm{C}-\mathrm{H} \\
\mathrm{Ar}\end{array}$} & \multirow{2}{*}{$\mathrm{C}=\mathrm{N}$} & \multirow{2}{*}{$\mathrm{N}-\mathrm{N}$} & \multirow{2}{*}{$\mathrm{C}=\mathrm{C}$} & \multicolumn{2}{|c|}{$\mathrm{C}-\mathrm{O}-\mathrm{C}$} & \multirow{2}{*}{ Other } \\
\hline & & & & & & & & As & Sy & \\
\hline 13 & $\mathrm{H}$ & 3587 & 3216 & 3072 & 1637 & 1027 & 1507 & 1236 & 1118 & \\
\hline 14 & $2-\mathrm{Cl}$ & 3574 & 3330 & 3090 & 1685 & 1029 & 1509 & 1240 & 1036 & (767)C-Cl \\
\hline 15 & $4-\mathrm{OCH}_{3}$ & 3574 & 3332 & 3093 & 1605 & 1020 & 1506 & 1249 & 1035 & \\
\hline 16 & $4-\mathrm{NO}_{2}$ & 3524 & 3166 & 3076 & 1695 & 1033 & 1508 & 1260 & 1033 & $\begin{array}{l}\mathrm{As}\left(\mathrm{C}-\mathrm{NO}_{2}\right)(1455) \\
\mathrm{Sy}\left(\mathrm{C}-\mathrm{NO}_{2}\right)(1336)\end{array}$ \\
\hline
\end{tabular}

Table (13): IR spectral data for compounds (17-20)

\begin{tabular}{|c|c|c|c|c|c|c|c|}
\hline \multirow{2}{*}{ Comp no. } & \multirow{2}{*}{$X$} & \multicolumn{6}{|c|}{ IR $v \mathrm{~cm}^{-1}(\mathrm{KBr})$} \\
\hline & & $\mathrm{O}-\mathrm{H}$ & $(\mathrm{C}-\mathrm{H})_{\mathrm{Ar}}$ & $\mathrm{C}=\mathrm{O}$ & $\mathrm{C}=\mathrm{N}$ & $\mathrm{N}-\mathrm{N}$ & Other \\
\hline 17 & $\mathrm{H}$ & 3612 & 3084 & 1635 & 1558 & 1426 & a \\
\hline 18 & $4-\mathrm{NO}_{2}$ & 3483 & 3084 & 1596 & 1558 & 1471 & $\begin{array}{c}\mathrm{As}\left(\mathrm{C}-\mathrm{NO}_{2}\right) \\
1508 \\
\mathrm{Sy} \\
\left(\mathrm{C}-\mathrm{NO}_{2}\right) \\
1361\end{array}$ \\
\hline 19 & $4-\mathrm{OCH}_{3}$ & 3499 & 3128 & 1646 & 1557 & 1507 & \\
\hline 20 & 2-Cl & 3480 & 3076 & 1620 & 1554 & 1446 & $(\mathrm{C}-\mathrm{Cl})(743)$ \\
\hline
\end{tabular}

Table (14): IR spectral for compounds (21-24)

\begin{tabular}{|c|c|c|c|c|c|c|c|}
\hline \multirow{3}{*}{$\begin{array}{l}\text { Comp } \\
\text { no. }\end{array}$} & \multirow{3}{*}{$X$} & \multicolumn{6}{|c|}{ IR v cm ${ }^{-1}(\mathrm{KBr})$} \\
\hline & & \multirow{2}{*}{$\mathrm{O}-\mathrm{H}$} & \multirow{2}{*}{$\mathrm{C}-\mathrm{H}_{\mathrm{Ar}}$} & \multirow{2}{*}{$\mathrm{C}=\mathrm{O}$} & \multicolumn{2}{|c|}{$\mathrm{C}-\mathrm{O}-\mathrm{C}$} & \multirow{2}{*}{ Other } \\
\hline & & & & & As & Sy & \\
\hline 21 & $4-\mathrm{NO}_{2}$ & 3177 & 3030 & 1651 & 1285 & 1130 & $\begin{array}{l}\mathrm{As}\left(\mathrm{C}-\mathrm{NO}_{2}\right)(1559) \\
\mathrm{Sy}\left(\mathrm{C}-\mathrm{NO}_{2}\right)(1340)\end{array}$ \\
\hline 22 & $4-\mathrm{OCH}_{3}$ & 3669 & 3348 & 1649 & 1287 & 1076 & \\
\hline 23 & $2-\mathrm{Cl}$ & 3496 & 3118 & 1640 & 1280 & 1154 & $\mathrm{C}-\mathrm{Cl}(749)$ \\
\hline 24 & $\mathrm{H}$ & 3315 & 3119 & 1653 & 1282 & 1168 & \\
\hline
\end{tabular}


Synthesis of Some Heterocyclic Compounds Derived from

1-Aryl-3-(4-hydroxy-3-methoxyphenyl) propene-1-one

Eman Ibrahim AL-Salihi Ahmad Khidr Ahmad

Table (15): IR spectral data for compounds (25-28)

\begin{tabular}{|c|c|c|c|c|c|c|c|c|c|}
\hline \multirow[t]{3}{*}{ Comp no. } & \multirow[t]{3}{*}{$\mathrm{X}$} & \multicolumn{8}{|c|}{ IR v cm ${ }^{-1}(\mathrm{KBr})$} \\
\hline & & \multirow[t]{2}{*}{$\mathrm{O}-\mathrm{H}$} & \multirow[t]{2}{*}{$\mathrm{C}-\mathrm{H}$} & \multirow[t]{2}{*}{$\mathrm{C}=\mathrm{N}$} & \multirow[t]{2}{*}{$\mathrm{C}=\mathrm{C}$} & \multirow[t]{2}{*}{$\mathrm{C}=\mathrm{S}$} & \multirow{2}{*}{$\mathrm{C}=\mathrm{\cdots} \mathrm{C}$} & \multicolumn{2}{|c|}{$\mathrm{C}-\mathrm{O}-\mathrm{C}$} \\
\hline & & & & & & & & As & Sy \\
\hline 25 & $4-\mathrm{OCH}_{3}$ & 3596 & 3212 & 1683 & 1637 & 1115 & 1601 & 1318 & 1036 \\
\hline 26 & $\mathrm{H}$ & 3466 & 3118 & 1636 & 1551 & 1166 & 1505 & 1291 & 1123 \\
\hline 27 & $2-\mathrm{Cl}$ & 3347 & 3128 & 1635 & 1557 & 1155 & 1539 & 1287 & 1071 \\
\hline 28 & $4-\mathrm{NO}_{2}$ & 3487 & 3218 & 1669 & 1636 & 1161 & 1577 & 1269 & 1124 \\
\hline
\end{tabular}

Table (16): IR spectral data for compounds (29-32)

\begin{tabular}{|c|c|c|c|c|c|c|}
\hline \multirow{2}{*}{ Comp no. } & \multirow{2}{*}{$\mathrm{X}$} & \multicolumn{5}{|c|}{$\mathrm{IR} \mathrm{v} \mathrm{cm}^{-1}(\mathrm{KBr})$} \\
\cline { 3 - 7 } & & $\mathrm{O}-\mathrm{H}$ & $\mathrm{C}-\mathrm{H}$ & $\mathrm{C}=\mathrm{N}$ & $\mathrm{N}-\mathrm{O}$ & Other \\
\hline 29 & $4-\mathrm{OCH}_{3}$ & 3272 & 3072 & 1654 & 750 & 784 \\
\hline 30 & $4-\mathrm{NO}_{2}$ & 3419 & 3079 & 1614 & $\begin{array}{r}\mathrm{As}\left(\mathrm{C}-\mathrm{NO}_{2}\right)(1505) \\
\mathrm{Sy}\left(\mathrm{C}-\mathrm{NO}_{2}\right)(1338)\end{array}$ \\
\hline 31 & $2-\mathrm{Cl}$ & 3420 & 3079 & 1624 & 775 & $821(\mathrm{C}-\mathrm{Cl})$ \\
\hline 32 & $\mathrm{H}$ & 3419 & 3072 & 1635 & 797 & \\
\hline
\end{tabular}

\section{$\underline{\text { References }}$}

1. M.J.Elarfi and H.A.Al-Difar ; Appl.Chem.42,6243-6245 (2012)

2. P.J.Jainey and K.I.ShwarBhat; Asian J of Bio. Chem. and Pharma. Res.3,2231-2560 (2013).

3. P. Sudhakar, and S. S. Bhale, "Organic Chemistry", 4(1), 35-39, (2014) (IVSL).

4. A. Kumar, J. Fernandes and P-Kumar, World Journal of pharmacy and pharmaceutical Sciences, 3(2), 1267-1277, (2014).

5. M.Verma, M.Tripathi, A.K. Saxena, Eur.J.Med.Chem. 29,941-949, (1994).

6. C.A.Winter, E.A.Risely, C.W.Nuss, ProcSocExp.Biol.Med., Vol.111, 544-547, (1962).

7. A. Deep, S-Jain and P. C. Sharma, Actapoloniaepharma-Drug Res, 67(1), 63-67, (2010).

8. A. Burger, "In Burgers Medicinal Chemistry and Drug Discovery" 5th ed. Practical and principal, 1970-2000. (2003).

9. C.Gopinath,R.Nadendla,Lakshmi,Vijaya, M.K.Prahllad, K.B.N.Rao, Journal of Global Trends in Pharmaceutical Sciences, 1(1), P.26-41, (2011). 


\section{Synthesis of Some Heterocyclic Compounds Derived from \\ 1-Aryl-3-(4-hydroxy-3-methoxyphenyl) propene-1-one}

\section{Eman Ibrahim AL-Salihi}

Ahmad Khidr Ahmad

10. N. Garg, T. Chandra, Archana, A. Jain, A. Kumar, European Journal of Medicinal Chemistry, 45, 1529-1535, (2010) (IVSL).

11. J . S. Yadar, Y. K. Srivastava, Der Pharmacia Letter, 3(2), 284-291, (2011) .

12. A . Levia, J . Jozesf, Arkivoc, 1, 134-145, (2007).

13. A . C. Rana, A . Singh, J. Chem. Pharm. Res, 2 (1), 505-511, (2010).

14. N. H. Cromwel, R. E . Bamlurg and R. P. Bakly, J. Am. Chem. 1

15. أيوب، مقداد توفيق ؛ زكريا ، مروان محمود ؛ سعيد ، عبدالقادر سعيد ؛ مجلة علوم الر افدين ، 11(4) ، 36-43 ،

.$(2000)$

16. S.Tammoto, Y.Maghraby,R.Oda. Jap Chem.Soc, Kyoto, Japan, 66,48-50, (1963).

17. P . C . Sharma. S . V . Sharma, S . Jain,D. Singh and B. Suresh,ActaPolinaePharmaceutice - Drug Research, 66 (1), 101-104,(2009).

18. A.Sehlinger,O.Kreye, ,M.A.R. Meier,. Tunable polymers obtained from passerinMulticompound reaction derived acrylate monomers. Macromolecules, (2013).

19. K.M.Amrit, G.Sujay, C.Suchandra, B.Samita,. Chandan, Journal of Luminescence, 143, 693-703, (2013).

$$
\begin{aligned}
& \text { 20. في ام بأرخ ، ترجمة الشربة ، عبد الحسين خضر ، الراوي، جاسم ، محمد عليالعر اقي ، محمد احمد " اطياف } \\
& \text { امتصاص الجزيئات العضوية " ص176، مديرية مطبعة جامعة الموصل،(1985). }
\end{aligned}
$$

21. I.R.Dim,A.Sahin,. Bioactive volatile content of the stem and root of centaureaCarduiformis DC. Subsp. Carduiformis var. Carduiformis .J. Chem, 10, 1-6, (2013).

22. V. M. Barot and B. G. Rathod, Asian Journal of Biochemical and Pharmaceutical Research Issue, 4 (2), (2012).

23. K.Shuna,N.Joseph,H.philippe and J. N. Vishwakarma" Organic Research Lab., Department of Chemical Sciences", Assam Don Bosco University, 4(1), 137-145, (2014)(IVSL).

24. V.L.Fleisher, M.V.Andryukhova Chemistry, Technology of Organic Substances Technology and Biotechnology, (2012). 